\title{
AVALIAÇÃO OFTALMOLÓGICA TARDIA EM PORTADORES DE RETINOPATIA DIABÉTICA
}

\author{
Otacílio de Oliveira Maia Júnior*, Roberta Ferrari Marback, Maria Teresa Brizzi Bonanomi, Walter Yukihiko Takahashi, Newton Kara-José
}

Trabalho desenvolvido no Departamento de Oftalmologia do Hospital das Clínicas da Faculdade de Medicina da Universidade de São Paulo (HCFMUSP), Setor de Retina e Vítreo

\author{
*Correspondência: \\ Rua Machado Bittencourt, \\ 29 , apto. 53. \\ Vila Clementino. \\ São Paulo/SP \\ Cep: 04044-000 \\ Tels: (11) 9128-6672 / (11) \\ 5084-2790 \\ omaiausp@uol.com.br
}

\begin{abstract}
RESUMO
Oвjetrvo. Analisar o retardo na avaliação oftalmológica em portadores de retinopatia diabética avançada e sem queixas visuais.

Métodos. Foi realizado um estudo transversal analítico com 44 portadores assintomáticos de retinopatia diabética. Todos os pacientes tiveram indicação de tratamento com laser em pelo menos um dos olhos. A duração e tipo da doença, bem como o tempo para a primeira avaliação oftalmológica foram determinados.

Resultados. Dos 44 pacientes, 6 (I3,7\%) eram portadores de diabetes mellitus tipo I e 38 (86,3\%) do tipo 2. Retinopatia diabética proliferativa estava presente em $24(54,5 \%)$ e retinopatia diabética não proliferativa grave ou muito grave em 20 (45,5\%). A acuidade visual, com melhor correção, pré-tratamento, variou de 0,5 a 1,0 (média de 0,83 $\pm 0,17$ ) na tabela de Snellen. 0 tempo de intervalo entre o diagnóstico de diabetes e a primeira avaliação oftalmológica variou de 7 a 19,5 anos (I3,3 $\pm 5, I$ anos) para os diabéticos tipo I e de 3 meses a I 8 anos (5,2 \pm 4,8I anos) para os tipo 2.

Conclusão. Retinopatia diabética avançada pode estar presente em diferentes momentos na avaliaçãa oftalmológica para ambos os tipos de diabetes. No presente estudo, embora a média de intervalo entre o diagnóstico de diabetes e a primeira avaliação seja de 13 anos para os diabéticos tipo I e de 5 anos para os tipo 2, a retinopatia avançada foi observada em até 3 meses após diagnóstico de diabetes. Este fato demonstra a necessidade de avaliação oftálmica mais precoce nesta doença. Dessa forma, conclui-se que a RD deve ter uma abordagem multidisciplinar, no intuito de que o diagnóstico da RD seja feito, em suas fases iniciais, antes que lesões comprometedoras da visão já tenham ocorrido.
\end{abstract}

UnItermos: Retinopatia diabética/terapia. Angiopatias diabéticas. Diabetes mellitus/complicações. Retinopatia diabética/prevenção e controle. Retinopatia diabética/diagnóstico. Saúde Pública.

\section{INTRODUÇÃO}

O diabetes mellitus (DM) é um dos principais problemas de saúde pública devido às complicações crônicas incapacitantes e ao aumento da mortalidade dos indivíduos afetados. Estima-se que, no Brasil, 7,6\% da população urbana entre 30 e 69 anos apresentem DM, sendo que $46 \%$ destes não sabem ser portadores, de acordo com censo realizado pelo Ministério da Saúde em conjunto com a Sociedade Brasileira de Endocrinologia, em 1989'. A Organização Mundial da Saúde (OMS) acredita que o número de portadores de DM no mundo, que foi estimado em 177 milhões no ano de 2000, atingirá cifra em torno de 300 milhões em 2025, atribuindo este crescimento ao aumento de sobrevida, disseminação de dietas inadequadas e ao sedentarismo, principalmente nos países desenvolvidos?

Uma das complicações microvasculares mais importantes do DM é a retinopatia diabética $(R D)$, que é a principal causa de cegueira entre norte-americanos na faixa etária entre 20 e 64 anos, causando 8000 novos casos de cegueira a cada ano ${ }^{3}$. No Brasil, estima-se que metade dos pacientes portadores de DM seja afetada pela $\mathrm{RD}$, sendo responsável por 7,5\% das causas de incapacidade de adultos para o trabalho!.

O Early Treatment Diabetic Retinopathy Study (ETDRS), estudo clínico randomizado multicêntrico, definiu as estratégias do tratamento da RD de acordo com a sua classificação ${ }^{4}$, bem como a técnica de aplicação recomendada ${ }^{5,6}$. Segundo este estudo, o laser focal ou em grade na mácula deve ser aplicado no edema macular clinicamente significativo ou no edema difuso, respectivamente ${ }^{5}$; a panfotocoagulação da retina é indicada para RD não proliferativa grave e muito grave e para RD proliferativa ${ }^{6,7}$. O ETDRS sugeriu que o tratamento precoce com laser reduz o risco de piora na acuidade visual (AV) em mais de $50 \%^{8}$. A AV não é parâmetro para indicação de tratamento da $\mathrm{RD}^{6,7}$.

O objetivo deste trabalho é analisar o retardo do exame oftalmológico em portadores assintomáticos de retinopatia diabética avançada e com indicação de tratamento, avaliando o tempo decorrido entre o diagnóstico de DM e o primeiro exame de fundo de olho e as implicações deste intervalo para eficácia da terapia. Este trabalho faz parte de pesquisa mais ampla acerca desta problemática.

\section{Métodos}

Foi realizado um estudo transversal analítico com portadores assintomáticos de retinopatia diabética. Foram incluídos 44 pacientes consecutivos que preencheram os seguintes critérios de inclusão: indivíduos de ambos os sexos, qualquer idade, portadores de retinopatia diabética avançada e com indicação da panfotocoagulação retiniana com laser, sem outro tipo de maculopatia ou queixas de diminuiçã̃o da acuidade visual. 
Tabela I - Resumo de características dos portadores de DM tipo 2 no momento da indicação de tratamento com laser. Hospital das Clínicas da Faculdade de Medicina da Universidade de São Paulo - 2005

\begin{tabular}{|c|c|c|c|c|c|c|}
\hline Paciente & Idade & Sexo & $\underline{\mathrm{Hb}_{\mathrm{Alc}}}$ & Classificação RD & Diagnóstico DM* & I a fundoscopia* \\
\hline 1 & 57 & $\bar{M}$ & 7.0 & RDP & 6.00 & 3.00 \\
\hline 2 & 51 & $\mathrm{~F}$ & 8,4 & RDP & 9,00 & 2,00 \\
\hline 3 & 54 & M & 10,7 & RDP & 4,00 & 3,00 \\
\hline 4 & 62 & $\mathrm{~F}$ & 7,8 & RDP & 7,00 & 3,00 \\
\hline 5 & 59 & M & 11,6 & RDP & 9,00 & 5,00 \\
\hline 6 & 46 & M & 6,8 & RDNP & 0,75 & 0,50 \\
\hline 7 & 63 & $\mathrm{~F}$ & 8,5 & RDNP & 20,00 & 2,00 \\
\hline 8 & 57 & M & 8,1 & RDP & 16,00 & 1,50 \\
\hline 9 & 65 & $\mathrm{~F}$ & 7,2 & RDNP & 15,00 & 3,00 \\
\hline 10 & 64 & $\mathrm{~F}$ & 10,5 & RDNP & 15,00 & 10,00 \\
\hline$\|$ & 54 & M & 10,2 & RDP & 20,00 & 10,00 \\
\hline 12 & 51 & $\mathrm{~F}$ & 10,7 & RDNP & 15,00 & 8,00 \\
\hline 13 & 47 & $\mathrm{~F}$ & 9,9 & RDNP & 16,00 & 16,00 \\
\hline 14 & 56 & $\mathrm{~F}$ & 8,1 & RDNP & 20,00 & 8,00 \\
\hline 15 & 60 & $\mathrm{~F}$ & 11,3 & RDP & 3,00 & 5,00 \\
\hline 16 & 54 & $\mathrm{~F}$ & 11,2 & RDP & 5,00 & 0,09 \\
\hline 17 & 66 & M & 8,2 & RDP & 7,00 & 0,50 \\
\hline 18 & 55 & $\mathrm{~F}$ & $\mid 2,1$ & RDNP & 7,00 & 0,75 \\
\hline 19 & 41 & M & 7,1 & RDP & 15,00 & 6,00 \\
\hline 20 & 47 & M & 6,6 & RDNP & 0,75 & 0,50 \\
\hline 21 & 59 & M & 8,5 & RDNP & 16,00 & 1,50 \\
\hline 22 & 52 & $\mathrm{~F}$ & 8,5 & RDP & 4,00 & 0,17 \\
\hline 23 & 55 & M & 11,3 & RDP & 10,00 & 8,00 \\
\hline 24 & 55 & M & 7,0 & RDNP & 4,00 & 3,00 \\
\hline 25 & 50 & M & 5,2 & RDP & 1,00 & 0,37 \\
\hline 26 & 40 & M & 8,0 & RDP & 24,00 & 16,00 \\
\hline 27 & 60 & M & 9,1 & RDP & 15,00 & 4,00 \\
\hline 28 & 43 & M & 10,1 & RDNP & 5,00 & 4,00 \\
\hline 29 & 58 & M & 8,1 & RDP & 15,00 & 15,00 \\
\hline 30 & 58 & $\mathrm{~F}$ & 9,3 & RDNP & 8,00 & 6,00 \\
\hline 31 & 50 & $\mathrm{~F}$ & 7,3 & RDNP & 12,00 & 5,00 \\
\hline 32 & 57 & M & 8,1 & RDP & 4,00 & 1,00 \\
\hline 33 & 49 & $F$ & 8,0 & RDNP & 7,00 & 3,00 \\
\hline 34 & 58 & $F$ & 8,9 & RDP & 10,00 & 8,00 \\
\hline 35 & 56 & M & 7,9 & RDP & 6,00 & 6,00 \\
\hline 36 & 54 & $\mathrm{~F}$ & 7,8 & RDP & 12,00 & 1,00 \\
\hline 37 & 65 & M & 8,5 & RDP & 8,00 & 4,00 \\
\hline 38 & 69 & M & 8,0 & RDNP & 6,00 & 2,00 \\
\hline
\end{tabular}

*Tempo (em anos) definido na indicação do tratamento; DM: diabetes mellitus; RD: retinopatia diabética; RDNP: retinopatia diabética não proliferativa grave ou muito grave; RDP: retinopatia diabética proliferativa

O tempo de diagnóstico e a primeira avaliação oftalmológica dos pacientes foram determinados. O controle sistêmico foi avaliado por uma medida isolada da hemoglobina glicosilada $\left(\mathrm{Hb}_{\mathrm{Al}}\right)$ (método certificado pelo National Glycohemoglobin Standardization Program (NGSP); valor de referência $=4,1 \mathrm{~A}$ $6,5 \%)$.

Os pacientes selecionados foram submetidos inicialmente a uma avaliação da AV com melhor correção (tabela de Snellen), fundoscopia e exames complementares: retinografia e angiofluoresceinografia. Após esta avaliação, foram programadas aplicações de laser conforme padronização recomendada pelo ETDRS.

As variáveis quantitativas foram expressas em médias e desvios padrão; com as qualitativas calculou-se freqüências absolutas e relativas. O estudo foi aprovado pela Comissão de Ética para Análise de Projetos e Pesquisa da Diretoria Clínica do Departamento de Oftalmologia do Hospital das Clínicas da Faculdade de Medicina da Universidade de São Paulo e todos os pacientes assinaram o termo de consentimento livre e esclarecido.

\section{Resultados}

O sumário das características dos pacientes está demonstrado nas Tabelas I e 2. A amostra foi formada por 44 pacientes, sendo 6 $(13,7 \%)$ portadores de DM tipo I e 38 (86,3\%) de tipo 2. A idade variou entre 24 e 69 anos, com média de 29 anos para os pacientes de DM tipo I e de 55,2 anos para DM tipo 2. A predominância foi do sexo feminino para os dois tipos de DM (66,7\% tipo I e 52,3\% tipo 2). 
Tabela 2 - Resumo de características dos portadores de DM tipo I no momento da indicação de tratamento com laser. Hospital das Clínicas da Faculdade de Medicina da Universidade de São Paulo - 2005

\begin{tabular}{|c|c|c|c|c|c|c|}
\hline Paciente & Idade & Sexo & $\mathrm{Hb}_{\mathrm{Alc}}$ & Classificação RD & Diagnóstico DM* & Ia fundoscopia* \\
\hline 1 & $\overline{24}$ & $\bar{F}$ & 8.9 & $\overline{R D N P}$ & $\frac{15,0}{15}$ & 6,0 \\
\hline 2 & 30 & M & 7,0 & RDNP & 20,0 & 1,0 \\
\hline 3 & 34 & $\mathrm{~F}$ & 7,9 & RDP & 20,8 & 8,0 \\
\hline 4 & 24 & $\mathrm{~F}$ & 8,0 & RDNP & 15,0 & 3,0 \\
\hline 5 & 34 & $\mathrm{~F}$ & 7,0 & RDP & 20,0 & 0,5 \\
\hline 6 & 28 & M & 9,0 & RDNP & 25,0 & 18,0 \\
\hline
\end{tabular}

*Tempo (em anos) definido na indicação do tratamento; DM: diabetes mellitus; RD: retinopatia diabética; RDNP: retinopatia diabética não proliferativa grave ou muito grave; RDP: retinopatia diabética proliferativa

A classificação da RD teve 24 pacientes (54,5\%) com RD proliferativa e 20 (45,5\%) com RD não proliferativa grave ou muito grave.

O tempo decorrido entre o diagnóstico de DM e o primeiro exame de retina sob midríase variou de 3 meses a 18 anos (média 5,2 \pm 4,8 I anos) para os portadores de DM tipo 2 e variou de 7 a 19,5 anos para o tipo I (média I3,2 \pm 5, I2 anos). A hemoglobina glicosilada ( $\mathrm{Hb}_{\text {Alc }}$ ) variou de 7 a 9 para os DM tipol (média 8,6 $\pm 0,87$ ) e de 5,2 a I2, I para os tipo 2 (média de 8,7 $\pm 1,60$ ).

A acuidade visual pré-tratamento variou de 0,5 a 1,0 (média de $0,83 \pm 0,17$ ). Todos os pacientes tiveram indicação de panfotocoagulação retiniana com laser em pelo menos um dos olhos.

\section{Discussão}

Estudos recentes sugerem diminuição na prevalência da RD. Uma maior eficácia no tratamento do DM (melhor controle da glicemia e da pressão sangüínea) $)^{9,10}$, diagnóstico mais precoce (por meio de programas de rastreamento e de redução na taxa glicêmica para definir o portador da doença) e avanços propedêuticos para examinar a retina são fatores que, possivelmente, estão associados a este decréscimo ${ }^{11-13}$. Assim, Brown et al. demonstraram que, com esta evolução, diabéticos do tipo 2 tiveram uma menor prevalência de RD incipiente quando comparados com o resultado do clássico estudo Wisconsin Epidemiologic Study of Diabetic Retinopathy (WESDR) 14,15; entretanto, não foi observada mudança na progressão para as formas avançadas da RD. Por outro lado, foi evidenciada que a prevalência da RD em diabéticos do tipo I é alta, sendo observado que $86 \%$ desses pacientes possuem algum grau de $\mathrm{RD}$ e que metade precisa ser submetida ao tratamento com fotocoagulaçãa ${ }^{16}$.

Em qualquer estágio da RD pode ocorrer diminuição importante da $A V$, causada pelo edema macular clinicamente significativo, o qual foi definido pelo ETDRS e consiste na maior causa de diminuição da AV na $\mathrm{RD}$, principalmente em pacientes com DM tipo $2^{5}$. A cegueira está associada à fase avançada da RD representada pela RD proliferativa e suas manifestações: hemorragia pré-retiniana ou vitrea (Figura I), proliferação fibrovascular e descolamento tracional de retina (Figura 2), neovasos de íris e glaucoma neovascular'. Em nosso estudo, 54,5\% dos pacientes tiveram a RD classificada como proliferativa, ainda sem apresentar as complicações supracitadas.

As medidas preventivas adequadas incluem, além do controle da glicemia e da pressão arterial, a realização do diagnóstico em uma fase inicial e passível de intervenção. Essas medidas diminuem a progressão das alterações retinianas, não revertendo os danos já
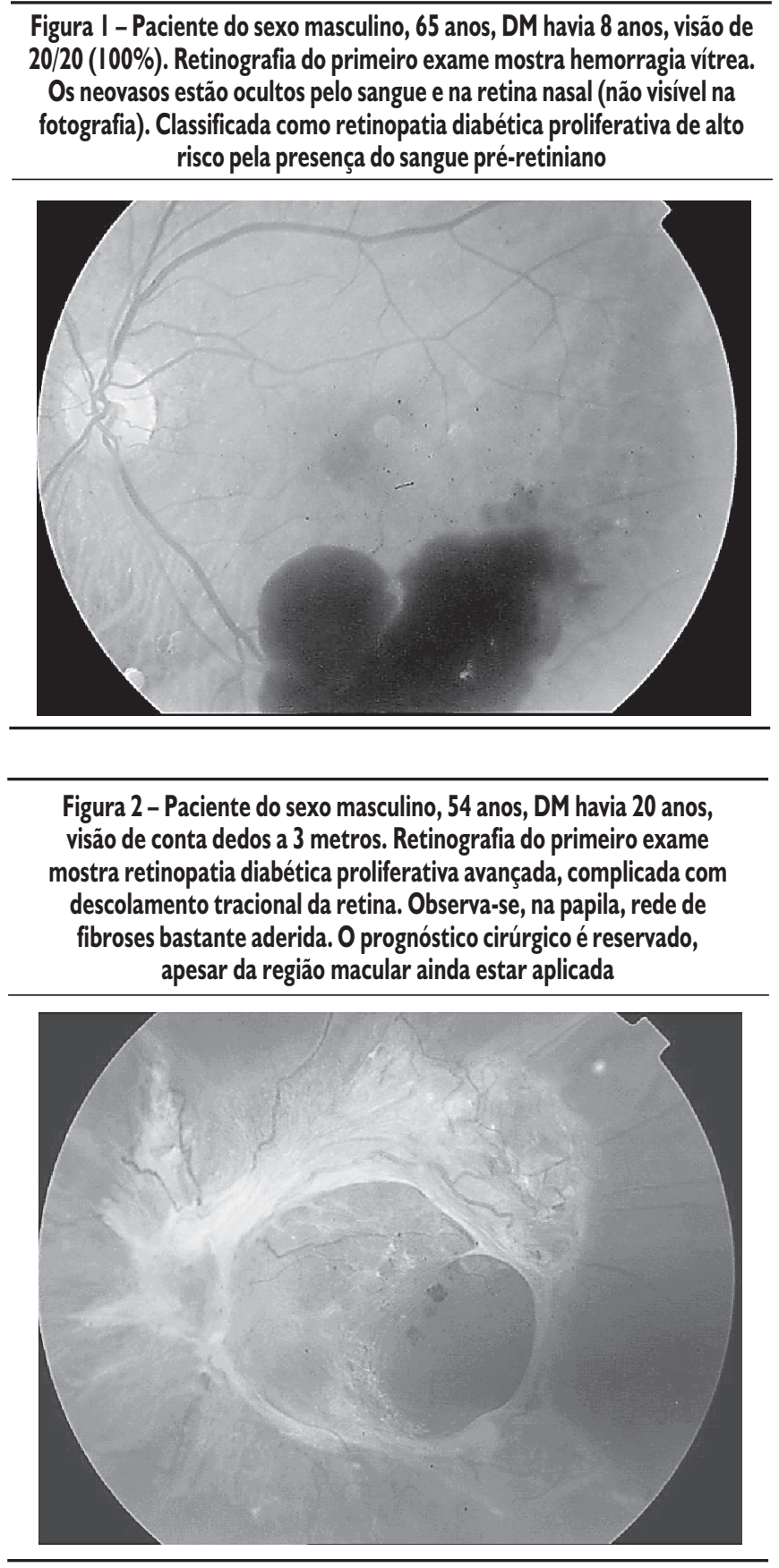
estabelecidos ${ }^{17}$. A associação $\mathrm{da} \mathrm{Hb}_{\mathrm{Alc}}>8,6 \% \mathrm{com}$ a taxa de progressão para RD proliferativa foi demonstrada recentemente ${ }^{18}$. No presente estudo, a média da $\mathrm{Hb}_{\mathrm{Alc}}$ foi mantida em nível de progressão da retinopatia.

Não existe cura para a RD. Os estudos multicêntricos realizados (DRS - Diabetes Retinopathy Study; ETDRS; DRVS - Diabetic Retinopathy Vitrectomy Study; DCCT - Diabetes Control and Complications Study; WESRD; UKPDS - United Kingdom Prospective Diabetic Study) estabeleceram a importância dos fatores de risco, seguimento e manejo da $R D^{19}$. Outras formas de tratamento estão sendo pesquisadas atualmente (acetato de triancinolona, antiangiogênicos, antiproliferativos); contudo, o tratamento estabelecido para a RD instalada é a fotocoagulação com laser de argônio, podendo evitar perda visual em casos selecionados e estabilizar a progressão da doença ${ }^{20}$.

Alguns estudos revelam que muitos diabéticos são avaliados e tratados de forma errônea e apenas $50 \%$ dos pacientes são examinados por oftalmologista adequadamente $21-23$. Dessa forma, perde-se o meIhor momento para iniciar o tratamento dos pacientes, que precede a baixa de AV ou outros sintomas visuais ${ }^{23}$. Infelizmente, devido ao fato de a baixa de $A V$ ser freqüentemente um sintoma tardio da $R D$, muitos pacientes permanecem sem diagnóstico, mesmo quando a doença já está causando lesões retinianas graves e irreversíveis ${ }^{24}$. Assim, o paciente é encaminhado ou procura tratamento apenas em fases avançadas da retinopatia ${ }^{25}$.

Para instituir uma efetiva aplicação de laser e prevenir eficazmente a cegueira, o tempo de detecção da RD é crucial $^{26}$. Observou-se neste estudo um retardo na avaliação oftalmológica inicial em todos os pacientes que, apesar de não apresentarem queixas visuais, tiveram indicação de panfotocoagulação retiniana com laser. O laser tem um papel fundamental no tratamento da $\mathrm{RD}$ e visa primordialmente a prevenção da perda visual. Na maioria dos pacientes, a aplicação do laser não melhora a visão já perdida. A dificuldade é que muitos pacientes são examinados tardiamente, perdendo-se assim a oportunidade de prevenir complicações importantes da $\mathrm{RD}^{24}$. Uma ação integrada entre o médico que oferece os primeiros cuidados aos pacientes recémdiagnosticados de DM e o oftalmologista torna-se imperiosa, podendo reduzir perda visual ${ }^{28}$.

A recomendação da Academia Americana de Oftalmologia é que todo diabético tipo 2 seja submetido a um exame de retina, sob midríase, no momento do diagnóstico, e do tipo I, após cinco anos do mesmo. Avaliações posteriores são orientadas anualmente ou mais precoces, a depender do estágio da $\mathrm{RD}^{27}$.

\section{Conclusão}

Retinopatia diabética avançada pode estar presente em diferentes momentos na avaliação oftalmológica em ambos os tipos de diabetes. No presente estudo, embora a média de intervalo entre o diagnóstico de diabetes e a primeira avaliação seja de 13 anos para os diabéticos tipo I e de 5 anos para os tipo 2, a retinopatia avançada foi observada em até 3 meses após diagnóstico de diabetes. Este fato demonstra a necessidade de avaliação oftálmica mais precoce nesta doença. Dessa forma, conclui-se que a RD deve ter uma abordagem multidisciplinar, no intuito de que o diagnóstico da $\mathrm{RD}$ seja feito, em suas fases iniciais, antes que lesões comprometedoras da visão já tenham ocorrido.

\section{Conflito de interesse: não há.}

\section{SUMMARY \\ Delay in ophthalmologic examination of diabetic RETINOPATHY PATIENTS}

OBJECTIVE. To assess the delay in the ophthalmic examination of patients affected with severe diabetic retinopathy but no visual complaint.

METHODS. A transversal analytical study was conducted of 44 visually asymptomatic diabetic patients. All were eligible to retinal panphotocoagulation in at least one eye. Duration and type of the disease as well as time of the first ophthalmic examination were established.

RESULTS. Of the 44 patients, 6 (13.7\%) had diabetes type I and 38 (86.3\%) had diabetes type 2. Proliferative diabetic retinopathy was present in 24 (54.5\%) and severe or very severe non proliferative retinopathy was present in 20 (45.5\%). Best visual acuity ranged from 0.5 to 1.0 (mean $0.83 \pm 0.17$ ) according to the Snellen chart. The time interval between diagnosis of diabetes and the first ophthalmic examination ranged from 7 to 19.5 years $(13.2 \pm 5$. I 2 ) for diabetes type 1 and from 3 months to 18 years (5.2 \pm 4.81 ) for diabetes type 2 .

CONCLUSION. Advanced diabetic retinopathy may be detected at different times of the ophthalmic examination in both types of diabetes. Although the mean interval between the diagnosis and the first examination is 13 years for diabetes type I and 5 years for diabetes type 2, this severe kind of retinopathy occurred as early as three months after diagnosis of diabetes. This points to the need for an earlier ophthalmic examination in the case of this disease. [Rev Assoc Med Bras 2007; 53(I): 39-43]

KEY WORDS: Diabetic retinopathy/therapy. Diabetic angiopathies. Diabetes mellitus/complications. Diabetic retinopathy/prevention \& control. Diabetic retinopathy/diagnosis. Public Health.

\section{REFERÊNCIAS}

I. Malerbi DA, Franco LJ. Multicenter study of the prevalence of diabetes mellitus and impaired glucose tolerance in the urban brazilian population aged 30-69 yr. Diabetes Care. 1992; I 5: I 509- 16.

2. World Health Organization. Diabetes: the cost of diabetes. Fact sheet $n^{\circ}$ 236. Revised September 2002 [online]. Available from: http:// www.who.int/mediacentre/factsheets/fs236/en/

3. American Academy of Ophthalmology Basic and Clinical Science Course.Section 12: Retina and vitreous. 1999-2000 [online] [cited mar 2003]. Available from: http://149.142.138.19/jseiweb/Education/ educ_educact_basicandclinical.htm

4. Early Treatment Diabetic Retinopathy Study Research Group: Fundus photographic risk factors for progression of diabetic retinopathy. ETDRS report no 12. Ophthalmology. 1991;98:823-33.

5. Early Treatment Diabetic Retinopathy Study Research Group .Treatment techniques and clinical guidelines for photocoagulation of diabetic macular edema. ETDRS report $n^{\circ} 2$. Ophthalmology. 1987;94:761-74.

6. Early Treatment Diabetic Retinopathy Study Research Group. Techniques for scatter and local photocoagulation treatment of diabetic retinopathy. ETDRS report 4. Int Ophthalmol Clin. 1987;27:265-72. 
7. Early Treatment Diabetic Retinopathy Study Group. Early Photocoagulation for diabetic retinopathy: ETDRS report number 9. Ophthalmology. 1991;98(Suppl 5):767-85.

8. ETDRS Research Group. Photocoagulation for diabetic macular edema. Early treatment diabetic retinopathy study report number I. group. Arch Ophthalmol. 1985; 103: 1 796-806.

9. Brown JB, Pedula KL, Summers KH. Diabetic retinopathy: contemporary prevalence in a well-controlled population. Diabetes Care. 2003;26:2637-42.

10. Ozmen B, Boyvada S. The relationship between self-monitoring of blood glucose control and glycosylated haemoglobin in patients with type 2 diabetes with and without diabetic retinopathy. J Diabetes Complications. 2003; 17:128-34.

I I. Klein R. Has the frequency of proliferative diabetic retinopathy declined in the US? Diabetes Care. 2003;26:269I-2.

12. Wong TY, Klein R, Islam FM, Cotch MF, Folsom AR, Klein BE, et al. Diabetic retinopathy in a multi-ethnic cohort in the United States. Am J Ophthalmol. 2006; 141:446-55.

13. Liu DP, Molyneaux L, Chua E, Wang YZ, Wu CR, Jing H, et al. Retinopathy in a Chinese population with type 2 diabetes: factors affecting the presence of this complication at diagnosis of diabetes. Diabetes Res Clin Pract. 2002;56: |25-3|

14. Klein R, Klein BE, Moss SE, Davis MD, DeMets DL. The Wisconsin epidemiologic study of diabetic retinopathy. II. Prevalence and risk of diabetic retinopathy when age at diagnosis is less than 30 years. Arch Ophthalmol. 1984; I 02:520-6.

15. Klein R, Klein BE, Moss SE, Davis MD, DeMets DL. The Wisconsin epidemiologic study of diabetic retinopathy. III. Prevalence and risk of diabetic retinopathy when age at diagnosis is 30 or more years. Arch Ophthalmol. 1984; 102:527-32.

16. Roy MS, Klein R, O' Colmain BJ, Klein BE, Moss SE, Kempen JH. The prevalence of diabetic retinopathy among adult type I diabetic persons in the United States. Arch Ophthalmol. 2004; | 22:546-5 |

17. Gross JL, Neheme M. Detecção e tratamento das complicações crônicas do diabetes melito: Consenso da Sociedade Brasileira de Diabetes e Conselho Brasileiro de Oftalmologia. Rev Assoc Med Bras. 1999:45:279-84.
18. Sato Y, Lee Z, Hayashi Y. Relationship between mean hemoglobin HbA Ic value and development of proliferative diabetic retinopathy. Jpn J Ophthalmol 200 I;45:523-7.

19. Boelter MC, Azevedo MJ, Gross JL, Lavinsky J. Fatores de risco para retinopatia diabética. Arq Bras Oftalmol. 2003;66:239-47.

20. Ávila M. A Retina do Séc XXI. Arq Bras Oftalmol. 2003;66:720-30.

21. Ganley JP. Erradication of blindness secondary to diabetic retinopathy. Epidemiology 1995;2:57-8.

22. Sprafka JM, Fritsche TL, Baker R. Prevalence of undiagnosed eye disease in high-risk diabetic individuals. Arch Intern Med. I 989; I 50:559-70.

23. Sinclair SH, Delvecchio C. The internist's role in managing diabetic retinopathy: screening for early detection. Cleve Clin J Med. 2004;7|: I $51-9$.

24. Nehemy MB. Retinopatia diabética. Revisão temática. Arq Bras Oftalmol. 1998:61:366-70.

25. Arrata KM, Waszak DMJ, Delai NR, Moreira Jr CA. Detecção precoce da retinopatia diabética: análise de 296 pacientes em um hospital universitário. Rev Bras Oftalmol 1996;55:63-9.

26. González VC, González VME, Martínez DS, Rivera MD, Arredondo PB, Islas AS, et al. A diabetic retinopathy screening program as a strategy for blindness prevention. Arch Med Res. 1997;28: 129-35.

27. Screening guidelines for diabetic retinopathy. American College of Physicians, American Diabetes Association and American Academy of Ophthalmology. Ann Intern Med. 1992; I | 6:683-5.

28. Sinclair SH; Delvecchio C. The internist's role in managing diabetic retinopathy: screening for early detection. Cleve Clin J Med. 2004;7|:151-9.

Artigo recebido: 04/10/05

Aceito para publicação: 07/08/06 